\title{
Lessons from CTF3
}

\section{Piotr K. Skowroński*}

CERN, Geneva, Switzerland

E-mail: Piotr.Skowronski@cern.ch

\section{Roberto Corsini}

CERN, Geneva, Switzerland

E-mail: Roberto.Corsini@cern.ch

The CLIC Test Facility (CTF3) was built to demonstrate the feasibility of the CLIC two beam acceleration scheme. The main issues to be verified were the high current drive beam generation using a fully loaded highly efficient linac and a beam combination scheme, based on transverse RF deflectors, to increase beam current and bunch repetition frequency. The drive beam has been used for GW level RF power production and two beam acceleration experiments. CTF3 was also a test ground for development of many accelerator technologies. Its operation was concluded in 2016 and in this contribution the results relevant for the CLIC design as well as for the whole accelerator physics community will be presented.

The European Physical Society Conference on High Energy Physics

5-12 July, 2017

Venice

*Speaker. 


\section{Introduction}

The CLIC International Collaboration built at CERN the CLIC Test Facility CTF3 (see Fig.1) in order to prove feasibility of the two-beam acceleration technology [1]. It consisted of a $150 \mathrm{MeV}$ electron linac, $42 \mathrm{~m}$ long Delay Loop (DL) and a $84 \mathrm{~m}$ Combiner Ring (CR). With help of RF deflector the delay loop was interleaving the $1120 \mathrm{~ns}$ beam pulse to produce four $140 \mathrm{~ns}$ pulses of double current, which were combined into single $140 \mathrm{~ns}$ pulse by the combiner ring. The recombined beam was delivered to the CLIC experimental area (CLEX) where it was decelerated to produce $12 \mathrm{GHz}$ RF power accelerating a probe beam. The probe beam was generated with a $200 \mathrm{MeV}$ injector (Concept d'Accélérateur Linéaire pour Faisceaux d'Electrons Sondes, CALIFES) located in the same area.

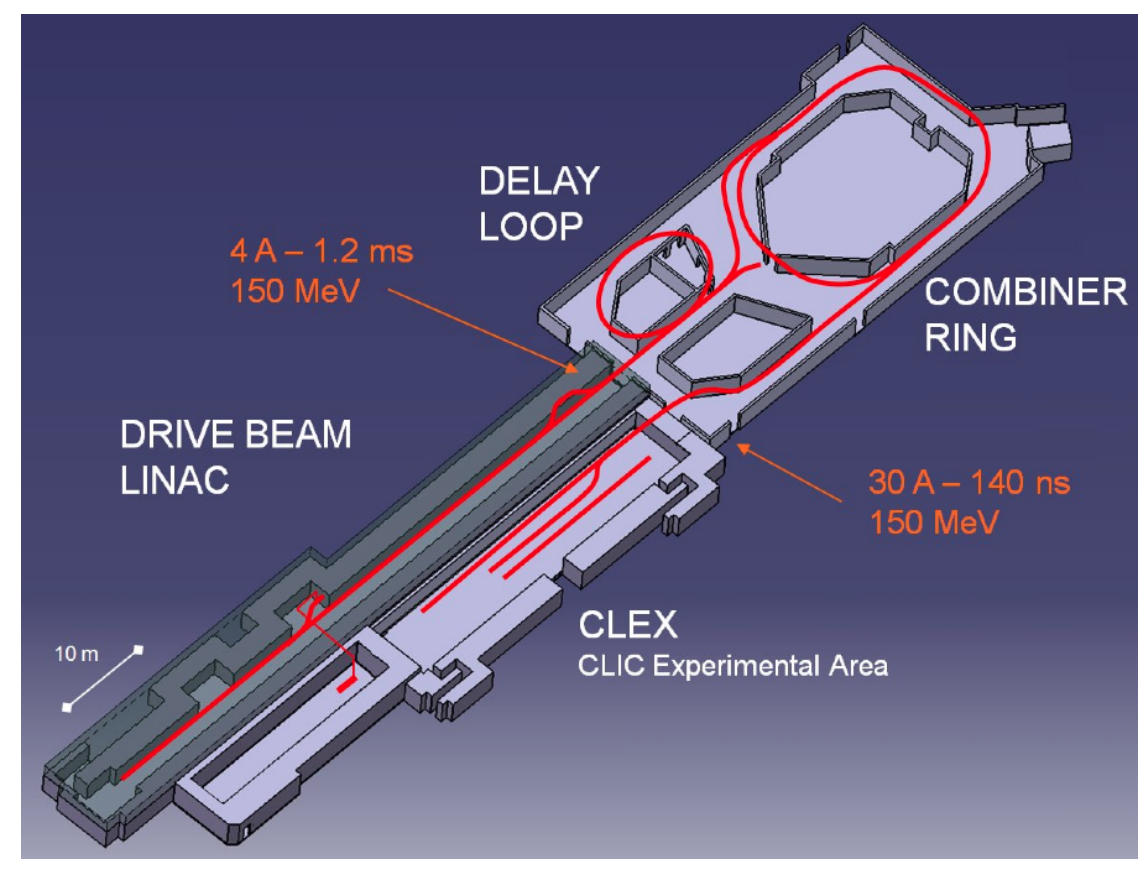

Figure 1: Schematic CTF3 layout.

The CTF3 studies can be divided into two categories [2]:

1. Efficient production of high-current electron drive beam with time structure suitable for $12 \mathrm{GHz}$ RF power production. CTF3 tested a novel technique based on fully-loaded acceleration in normal conducting travelling wave structures followed by beam current and bunch frequency multiplication in a series of delay lines and rings. CTF3 was producing in this way a 28 A electron beam with $12 \mathrm{GHz}$ bunch repetition frequency.

2. RF power production and two-beam acceleration. RF power is produced by decelerating the drive beam in dedicated cavities called PETS (Power Extraction and Transfer Structures). Using waveguides it is delivered to accelerating structures operating at gradient of $100 \mathrm{MV} / \mathrm{m}$. Deceleration studies were performed using a string of PETS in the Test Beam Line (TBL) in CTF3 experimental area (CLEX). Alternatively, the drive beam was delivered 
to another beam line called Two Beam Test Stand (TBTS) renamed later Test Beam Module (TBM), where one or more PETS powered one or more structures, further accelerating a $200 \mathrm{MeV}$ electron beam provided by CALIFES.

CTF3 was constructed and commissioned in stages. The linac started operation in 2003, the delay loop in 2006 and the combiner ring in the following year. In 2009 CLEX beam lines, including the CALIFES injector, and the transfer line from the combiner ring were commissioned. Recombination with the DL and CR together was achieved in autumn of 2009 yielding up to $28 \mathrm{~A}$ of beam current. In 2010 the PETS started producing the nominal power level and the first twobeam test was performed, reaching a measured gradient of $100 \mathrm{MV} / \mathrm{m}$. Ultimately, accelerating gradient of $145 \mathrm{MV} / \mathrm{m}$ was reached in 2011. The same year the PETS On-off mechanism was successfully tested. A $2 \mathrm{~m}$ long fully representative unit of the CLIC main linac called Two-Beam Module, TBM, replaced the TBTS at the end of 2014. In 2015 TBL decelerated the drive beam by $50 \%$ of its initial energy. The Drive beam stability and the overall performances of the facility were continually improved after the initial commissioning, until the final run in 2016. During the last years of operation CTF3 hosted several additional experiments and beam tests that were not in the initial planning. Most of them - but not all - were CLIC related.

\section{Drive Beam generation}

\subsection{Injector - Beam Current and Time Structure}

The drive beam injector consisted of a thermionic gun delivering up to $10 \mathrm{~A}$ beam, three $1.5 \mathrm{GHz}$ sub-harmonic bunchers (SHBs) and a $3 \mathrm{GHz}$ system composed of a pre-buncher and a buncher [3]. SHBs were 6-cell travelling wave (TW) structures with a nominal power of $40 \mathrm{~kW}$. They generated the initial energy-time modulation and phase coding by means of fast $180^{\circ} \mathrm{RF}$ phase switches. Following, a $3 \mathrm{GHz}$ single-cell pre-buncher and a TW buncher were installed to create the final bucket structure and accelerate the beam up to $\sim 6 \mathrm{MeV} / \mathrm{c}$. The nominal power of $2 \mathrm{~cm}$ long pre-buncher was $100 \mathrm{~kW}$, while for the half-meter long buncher it was maximum $40 \mathrm{MW}$.

The optimization of the transverse emittance, the bunch length and the satellite population was carried out by means of exhaustive PARMELA simulations. The magnetic field distribution was optimized to keep the emittance at the exit of the injector below $50 \mu \mathrm{m}$, which was confirmed by measurements [4]. A streak camera at the end of the linac measured a bunch length of $1 \mathrm{~mm}$ [5]. Some electrons were forming unwanted satellite bunches in between the main $1.5 \mathrm{GHz}$ ones. The measured fraction of the satellites was about $8 \%$, compared to the design value of $7 \%$. Figure 2 presents a projection of a streak camera image that shows the bunch population in function of time during the $180^{\circ}$ phase switch. The switching time is less than $6 \mathrm{~ns}$ (eight $1.5 \mathrm{GHz}$ periods), which is well below the $10 \mathrm{~ns}$ target [6].

\subsection{Linac - Full Beam-Loading Acceleration}

The overall efficiency is one of the key parameter of linear colliders. Full beam-loading operation is one of the key points in the CLIC design because it yields a very efficient RF energy transfer to the drive beam. It is achieved by the acceleration of a high beam current (about $4 \mathrm{~A}$ ) by short travelling-wave RF structures with relatively low gradient. When the beam is present in the cavity 


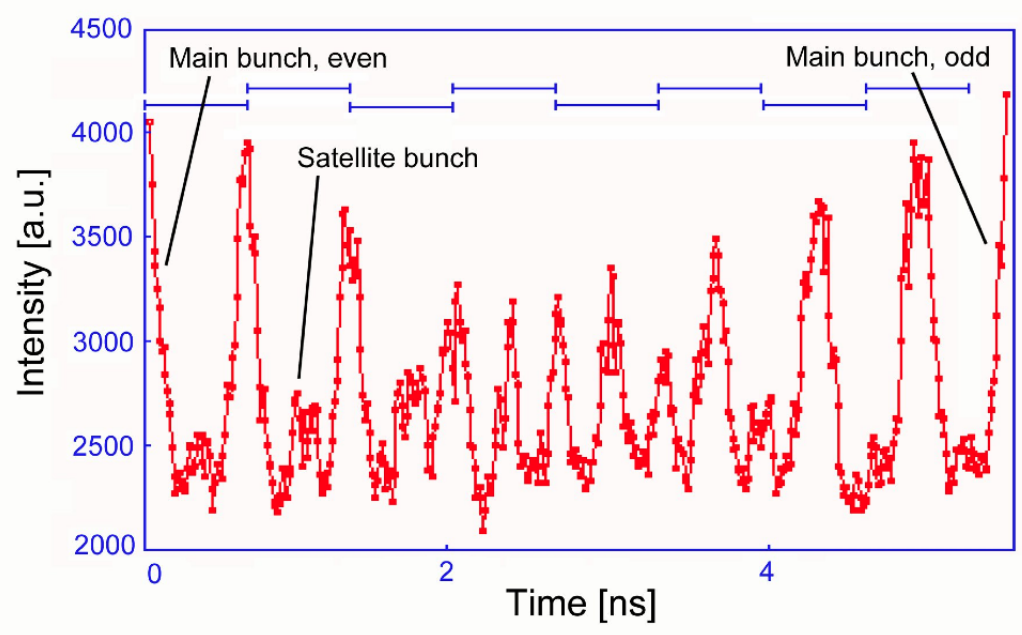

Figure 2: Fast bunch phase switch, measured by a streak camera. At the top the $1.5 \mathrm{GHz}$ periods are shown.

no RF power is transmitted to the load and the resistive losses in the walls are minimal. In the case of the CLIC structures an overall efficiency of about $98 \%$ is achieved. However, at the beginning of the pulse there is an energy transient effect, such that the first bunches may have twice the energy of the steady-state part. This mode of operation also strongly couples beam current fluctuations to the beam energy. The CTF3 $3 \mathrm{GHz}$ TW accelerating structures worked in the $2 \pi / 3$ mode. They were $1.22 \mathrm{~m}$ long and operated at a loaded gradient (nominal current) of $6.5 \mathrm{MV} / \mathrm{m}$ [7]. The large average current leads to pronounced effects of transverse higher order modes (HOMs). They must be damped in order to avoid beam instabilities and emittance growth. Dedicated cavities called Slotted Iris - Constant Aperture (SICA) structures were designed for this purpose. They feature irises that are radially slotted to guide dipole and quadrupole modes into $\mathrm{SiC}$ loads. The damped modes are selected through their field distribution, strongly damping the HOMs (Q factor typically below 20), while monopole modes are not influenced. Nose cones of variable geometry yield additional HOM detuning along the structure. This improves their suppression and modulates the group velocity to control the gradient profile. Thanks to the aperture that is kept constant along the structure short range wake-fields are reduced. The structures were powered by $35 \mathrm{MW}$ to $45 \mathrm{MW}$ klystrons providing $5.5 \mu \mathrm{RF}$ pulses. Each unit was powering 2 accelerating structures and the pulses were compressed to $1.3 \mu$ s pulses and over $60 \mathrm{MW}$. The pulse compression system uses a programmed phase ramp to provide a constant RF power.

The design beam current and pulse length were rapidly established during beam commissioning in June 2003. The beam was remarkably stable and no sign of beam break-up was observed at high current. It proved for the first time the fully loaded beam operation [8]. As predicted by simulations the normalized emittance at the end of the CTF3 linac was about $\varepsilon_{x, y} \approx 10 \mu \mathrm{m}$ confirming negligible effects of the wake-fields. The energy spread of the initial beam transient ( 100 ns) could also be reduced to a few percent by partial RF filling of the structures at beam injection.

The beam-to-RF phase was adjusted by maximizing the beam loading. It was achieved by minimizing the RF amplitude observed at the structure output couplers. The acceleration efficiency 
was assessed in a dedicated experiment [9]. The beam current and RF power measurements were carefully calibrated to compute the beam energy gain. It was then compared with the measurements using a spectrometer. Figure 3 shows that the RF power is almost fully absorbed by the beam. The energy gain measurements were in excellent agreement with the computed values. The obtained RF-to-beam transfer efficiency was $95.3 \%$ including the resistive losses. CTF3 successfully proved the stable and highly efficient acceleration of the drive beam as it was routinely operated over several years with fully loaded structures.

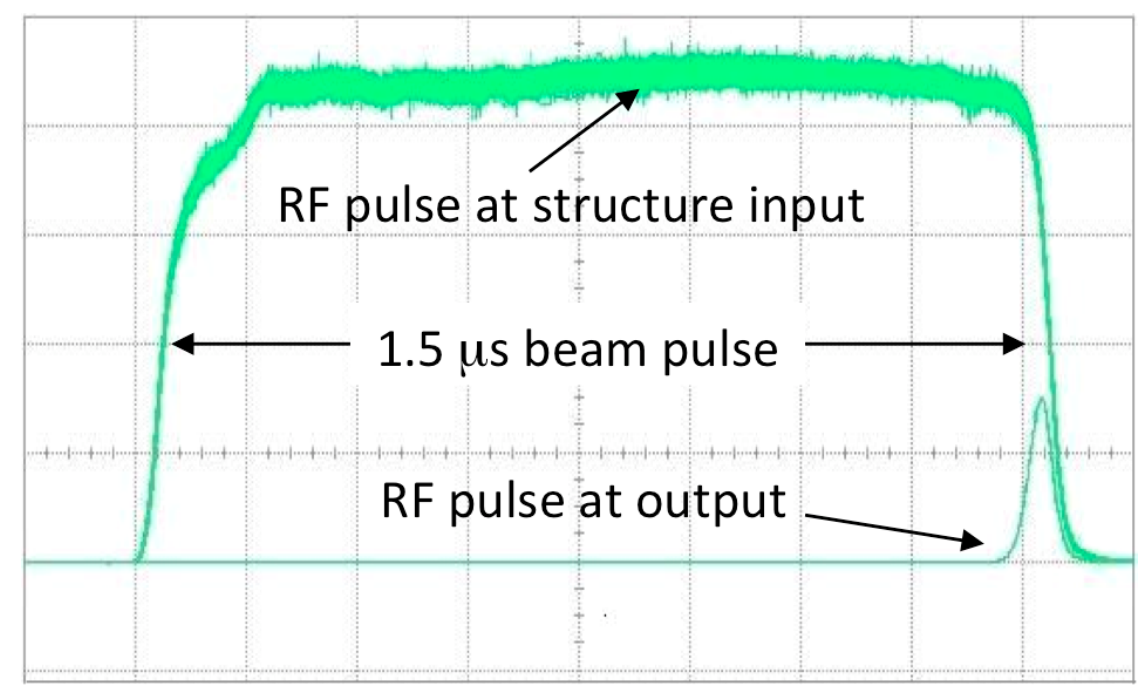

Figure 3: RF power measured at the accelerating structure input and output with beam.

\subsection{Delay Loop and CR - Bunch Combination}

CTF3 performed the beam recombination in two stages. First, the Delay Loop (DL) converted a $1120 \mathrm{~ns}$ long bunch train with a current of $\sim 4 \mathrm{~A}$ into 4 pulses of $140 \mathrm{~ns}$ and $~ 7.5 \mathrm{~A}$ (not counting the charge contained in satellite bunches). Later, the Combiner Ring (CR) interleaved these 4 pulses to produce a single $140 \mathrm{~ns}$ long pulse with a maximum current of about $30 \mathrm{~A}$.

The first RF deflector, operating at $1.5 \mathrm{GHz}$, sent odd phase-coded sub-pulses to the DL and the even ones straight towards the CR. The sub-pulse length must correspond to the length of the DL. The bunches leaving the DL were coming to the deflector at half of the wavelength distance from the bunches of the following even sub-pulse. Their orbits were merged obtaining $140 \mathrm{~ns}$ long pulses with twice the initial current and twice the bunch repetition frequency. In the CR a pair of RF deflectors created a time-dependent closed bump at injection to interleave the sub-trains.

The combination process must preserve transverse and longitudinal beam emittances: isochronous lattices, smooth linear optics, low impedance vacuum chambers and diagnostics, HOM free RF active elements are all needed to accomplish this task. Efficient RF power production in the PETS requires a short bunch length. The bunches get elongated if $R_{56}$ transfer matrix element is non zero. Therefore the bunch length preservation requires the use of isochronous optics $\left(\left|R_{56}\right| \leq 1 \mathrm{~cm}\right)$ in the DL, the CR and the transfer line connecting them. The CR layout was based on the use of three-dipole isochronous cells with three independent quadrupole families, whose tunability range 
fits well the requirements. The DL loop was made out of 2 six-dipole cells with eight independent quadrupoles. Sextupoles could be used to control the second-order matrix term $R_{566}$. After the linac the bunch length was less than $1 \mathrm{~mm}$ r.m.s. In CLEX a bunch length of $\sim 2 \mathrm{~mm}$ r.m.s. was estimated from RF power production and confirmed by direct streak camera measurements. Such length was consistent with required isochronicity conditions and entirely sufficient for CTF3 operation, avoiding also coherent synchrotron radiation, which would affect shorter bunches.

In the RF deflectors of the CR wake-fields in the vertical plane were minimized by detuning and damping [10]. The operational mode, i.e. the lowest order horizontal dipole one, naturally could not be damped or detuned. The turn-by-turn direct wake-field build-up was avoided because fill-time of the travelling wave deflectors was short enough ( $100 \mathrm{~ns})$. The residual amplification of the orbit errors by wake-fields was minimized by setting the fractional tune of the CR to about 0.6 in both planes. For the same reason the beam optics was chosen to minimize the beam size in the deflectors.

The recombination requires that the ring length is $\left(N \pm 1 / N_{f}\right) \lambda_{R F}$, where $\mathrm{N}$ is an integer number, $N_{f}$ the combination factor (here 4), and $\lambda_{R F}$ is the RF wavelength. The fractional part $\lambda_{R F} / N_{f}$ was measured precisely by Fourier transform of the beam phase monitor signal. The ring length was adjusted using a dedicated wiggler which could elongate the trajectory up to $7 \mathrm{~cm}$.

Demonstrating the very high beam quality required for CLIC was an integral part of the CTF3 experimental program. In the last years of the machine operation special effort was put into the drive beam performance optimization. The control of emittance growth, availability and stability was particularly improved. Emittance preservation required a good control of the optics, a very good closure of the DL and CR orbits, proper matching from the linac and control of spurious dispersion. The isochronous optics in DL, CR and the transfer lines were relatively strongly focusing. This, combined with a r.m.s. energy spread of about $0.6 \%$, lead to a large nonlinear dispersion, which was the main source of emittance growth. The most important growth was in the DL, whose optics was particularly constrained by building space limitations. A new DL optics, which was optimized to reduce the growth, was commissioned. Also tools for more precise measurements of Twiss parameters and dispersion in the different beam-lines were developed. The spurious dispersion was minimized with help of Dispersion Free Steering and Dispersion Target Steering procedures. While the beam combined only by the CR met the CLIC emittance requirements of $<150 \mu \mathrm{m}$ in both planes, the minimum horizontal emittance for the full factor 8 recombined drive beam was about $250 \mu \mathrm{m}$ ( $100 \mu \mathrm{m}$ in vertical). Besides demonstrating the feasibility of the CLIC bunch combination principle, CTF3 has allowed us to develop an optimized setting-up procedure of such a process, validating also the special diagnostics needed [11].

\subsection{Beam Stability Issues}

The CLIC two-beam acceleration scheme imposes tight tolerances on the stability of the drive beam current, energy and phase. A maximum variation of $0.75 \cdot 10^{-3}$ for the drive beam current and about $2^{\circ}$ at $12 \mathrm{GHz}$ for the drive beam bunch phase after combination are allowed. This is because they contribute quadratically to the luminosity loss [12] and these values correspond to a maximum of $1 \%$ to the luminosity loss per parameter. This already assumes a feed-forward system - discussed later - capable of reducing the phase jitter to $2^{\circ}$ at $12 \mathrm{GHz}$. 
During the initial years of operation CTF3 suffered from relatively large beam jitters and drifts. Dedicated studies identified their sources, which were either removed or compensated by proper feedback systems [15]. In the final CTF3 runs, experiments aiming at demonstrating performances close to the CLIC requirements were added to the CTF3 program. For the CTF3 klystrons the short-term RF stability over 500 consecutive RF pulses ( $\approx 10 \mathrm{~min}$.) was measured within the CLIC tolerances, for instance. The CLIC requires r.m.s. phase jitter below $0.05^{\circ}$ and r.m.s. amplitude jitter below $0.2 \%$ [12]. In CTF3 the mean pulse-to-pulse phase jitter measured with respect to an external reference was $0.035^{\circ}(3 \mathrm{GHz})$. The relative pulse-to-pulse amplitude jitter has been $0.21 \%$.

The pulse-to-pulse current variations in the CTF3 linac were measured using inductive beam position monitors. Initially it was $\Delta I / I=2 \cdot 10^{-3}$. It was improved by better stabilizing the gun heater power supply and by a feedback, to obtain $\Delta I / I=0.54 \cdot 10^{-3}$ [13], better than the required current stability for CLIC of $\Delta I / I=0.75 \cdot 10^{-3}$. After further improvements, in 2016 the r.m.s. current jitter at the end of the drive linac was at the level of the electronic noise floor of the BPMs 2 . $10^{-4}$, meaning that the observed jitter was the same with and without the beam. Such a performance was achieved for periods of tens of hours [14]. The stability of the combined beam current in CLEX, at the end of the experimental lines, was also largely improved and $3 \cdot 10^{-3}$ r.m.s. was measured for periods longer then 5 hours [15].

\subsection{The Drive Beam Phase Feed-Forward}

To assure the required drive beam timing stability below 50 fs r.m.s., or equivalently a phase stability (jitter) below 0.2 degrees of $12 \mathrm{GHz}$, CLIC will be equipped with a 'phase feed-forward' system. This system poses a significant challenge in terms of bandwidth, resolution and latency. Therefore, a prototype was designed, installed and tested in CTF3. After one year of experience a phase jitter of $0.28 \pm 0.02$ has been demonstrated, very close to the CLIC specifications [16]. With additional fine-tuning in 2016, the final year of operation at CTF3, it was possible to further reduce the achieved phase jitter to below the CLIC requirement of $0.2^{\circ}$ and to keep it at such level on time scales of about 10 minutes [17].

\section{Two-beam acceleration}

\subsection{Power generation, PETS On-Off}

In CTF3, PETS prototypes were tested with beam in TBL and in TBTS (and later TBM). The structures in the two lines had different lengths and a little bit different construction. The nominal CLIC parameters for power and pulse length were reached and exceeded for both of them. Maximum RF power reached at the nominal pulse length of $240 \mathrm{~ns}$ was about $300 \mathrm{MW}$, well above the nominal value for CLIC, $135 \mathrm{MW}$ [18].

Another CLIC feasibility verification was the PETS On-Off mechanism. It switches off individual PETS whenever localized breakdowns threaten the normal machine operation. The system also needs to provide a gradual ramp-up of the generated power in order to reprocess afterwards either the main accelerating structure and/or the PETS itself. The prototype is a compact external extension to PETS consisting of two high power variable RF reflectors. It was extensively tested 
with beam in the TBTS and the switching off of the PETS power production has been demonstrated with different beam and RF settings, up to 16 A and 200 MW [19]. The measurement of all the RF signals were in remarkable agreement with the model predictions in all conditions. When it is set to off the accelerating structure receives less than $1 \%$ of the power what makes any breakdowns impossible. Also the power inside PETS is attenuated to $\sim 25 \%$ of the nominal value what reduces the likelihood of a breakdown by a factor 102-103.

\subsection{Acceleration, Two-Beam Module}

The key purpose of CTF3 was to demonstrate the CLIC two-beam acceleration scheme. An efficient power transfer to high-gradient structures and acceleration of a "probe" electron beam is the heart of this technology. In CLEX, along the drive beam, a probe beam accelerator was installed delivering single bunches or bunch trains at $1.5 \mathrm{GHz}$ bunch repetition rate and energies up to $200 \mathrm{MeV}$. It consisted of a $24 \mathrm{~m}$ long electron injector linac CALIFES with $~ 5 \mathrm{MeV} / \mathrm{c}$ RF photocathode source [20]. The beam was accelerated in three $3 \mathrm{GHz}$ accelerating structures recuperated from the LEP Injector Linac (LIL). A single $3 \mathrm{GHz}$ klystron delivering $45 \mathrm{MW}$ RF pulses during $5.5 \mu$ sia an RF pulse compressor powered the accelerating structures and the photo-injector. CALIFES was usually operated with bunch charges of around $0.1 \mathrm{nC}$ and a normalized r.m.s. emittance of $10 \mu \mathrm{m}$. The probe beam energy was measured in a spectrometer in function of the probe beam $3 \mathrm{GHz}$ RF phase, which was phase-locked to the laser pulse timing. To adjust the relative timing between probe and drive beam for maximum acceleration phase scans were performed. The nominal CLIC accelerating gradient of $100 \mathrm{MV} / \mathrm{m}$ corresponded to an energy gain of $\Delta \mathrm{E}=$ 21.4 MeV. Energy gains of up to $\Delta \mathrm{E}=32 \mathrm{MeV}$ were reached with breakdown rates of a few $10^{-3}$ ), while higher gradients, up to $165 \mathrm{MV} / \mathrm{m}$ were achieved with higher rates [18]. Figure 4 shows an example measurement of probe beam acceleration using a spectrometer screen. The value $\Delta \mathrm{E}=$ $31 \mathrm{MeV}$ corresponds to an accelerating gradient of $145 \mathrm{MV} / \mathrm{m}$. The effect of breakdown kicks on the probe beam was also extensively studied [21].

At the end of 2014 in place of the TBTS the Two-Beam Module, TBM, a 2 m long fully representative unit of the CLIC main linac, including an active alignment system, was installed. The module was conditioned to the nominal CLIC gradient/pulse length and extensive tests of the module functionality were carried out. This included precision and reliability studies of the active alignment system and measurements of transverse wake-field effects. A full analysis of the experimental results is still undergoing. So far all the results are consistent with specifications and simulations.

\subsection{The Test Beam Line, TBL}

The Test Beam Line (TBL) served to study the stability of the drive beam during deceleration. It consisted of a FODO lattice with 14 consecutive PETS installed in the drift spaces. Precise beam alignment was possible thanks to high precision BPM's and quadrupole active movers. 25 A drive beam was decelerated from it initial energy of $135 \mathrm{MeV}$ down to a minimum of $67 \mathrm{MeV}$. This corresponds to the $50 \%$ deceleration milestone, which was one of the initial goals of CTF3. On average each PETS produced $90 \mathrm{MW}$ of power and the total peak RF power produced in less then $20 \mathrm{~m}$ deceleration line reached $1.3 \mathrm{GW}$. 


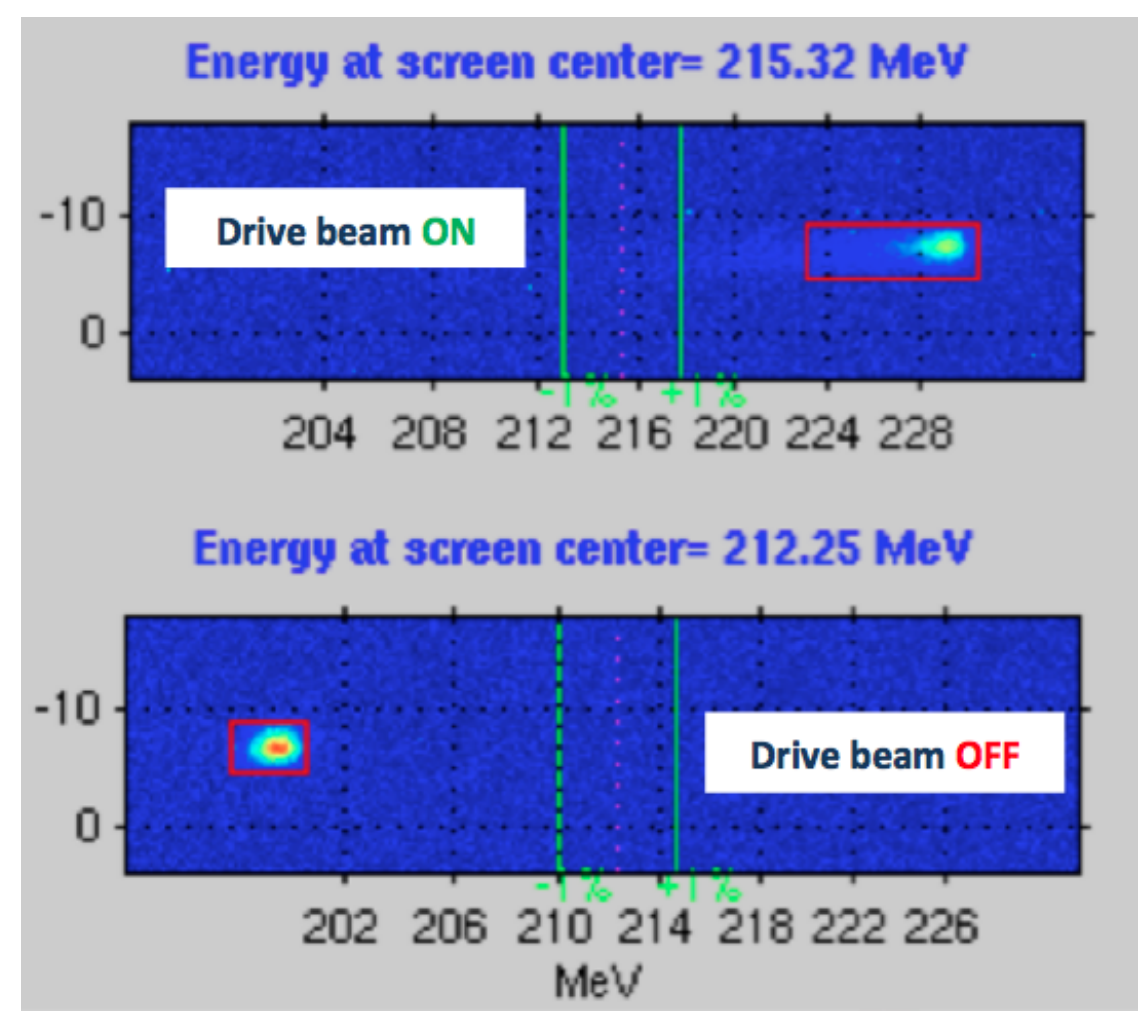

Figure 4: Probe Beam observed in the TBTS spectrometer screen with the $12 \mathrm{GHz}$ RF power from the drive beam on (top) and off (bottom). The energy gain is about $31 \mathrm{MeV}$, corresponding to a gradient of $145 \mathrm{MV} / \mathrm{m}$.

The dynamics of drive beam undergoing deceleration in the transverse planes was studied in detail. At the beginning and end of the line a time-resolved spectrometer provided measurement of the beam energy loss and its energy spread. Transverse Twiss parameters were measured with quadrupolar scans. Both longitudinal and transverse parameters agree very well with the simulations [22].

\section{Other experimental tests in CTF3}

\subsection{The Beam-Loading Experiment}

The RF breakdown rate (BDR) is critical for the luminosity performance of the CLIC linear collider. High power RF testing of a number of $12 \mathrm{GHz}$ CLIC prototype structures demonstrated rates of $10^{-7}$ at the design gradient of $100 \mathrm{MV} / \mathrm{m}$. Nevertheless, beam-loading significantly changes the field profile in the structures, and potential effects need to be understood. A dedicated experiment using the CTF3 drive beam was setup and data for different beam-loading configurations were collected in between 2014 and 2016 [23]. In some cases a BDR reduction up to an order of magnitude was measured. The results support the hypothesis of the BDR being dominated by the peak gradient and its reduction being associated to the modification of the gradient profile induced by the beam. Also, the measured distribution of the breakdowns inside the structure follows roughly the gradient profile, which additionally confirms this hypothesis. Therefore, a further 
optimization of the CLIC structure is possible by profiling the gradient such that distribution of breakdowns along the structure during the operation with beam is constant.

\subsection{Beam Diagnostics Tests}

An extensive test program for the main CLIC beam instrumentation components was established in CTF3 in order to verify the performance of the prototypes in a realistic environment. These devices need to provide state-of-the-art performance, or above. In many cases their number is such that cost-effective solutions are mandatory. Additionally, they need to detect tiny signals in the direct vicinity of hundreds MW RF power and operate in highly radioactive environment.

Wake Field Monitors (WFM) detects particular HOMs excited by the beam in the CLIC accelerating structures. HOM signals are picked up by 4 waveguide antennas attached to one of the structure cells. Two different frequency bands are analysed to measure the beam offset with respect to the structure with a required resolution of $3.5 \mu \mathrm{m}$. Two slightly different versions were tested in CTF3, and both demonstrated performances better or close to the required ones [24, 25].

Prototypes of drive beam BPM were also tested yielding $2 \mu \mathrm{m}$ resolution. However, the performance of the initial version was largely degraded in presence of $12 \mathrm{GHz}$ RF power from the drive beam. An improved design was successfully tested in 2015 [26]. Also, the cavity BPMs of the main linac initially showed worse than required time resolution when the position resolution was below specifications. Stainless steel was replaced with copper as the building material of the cavity and the Q value was optimized. The improved version showed a resolution below $1 \mu \mathrm{m}$, encouraging but still quite far from requirements [27].

At CLEX, along TBL and TBM lines, a cost effective optical fibre Beam Loss Monitor (BLM) system was installed. The system was successfully commissioned and various verification tests were performed. The provoked losses along the accelerator lines were localised by the BLMs with the expected accuracy below $2 \mathrm{~m}$, within CLIC requirements [28, 29]. Also, the amplitude of the signals agreed very well with conventional ionization chambers.

As a part of development of non-destructive profile monitors based on diffraction radiation, optical transition radiation interference was studied. In particular, for the first time shadowing effects were measured in imaging conditions, confirming the theoretical predictions [30].

\section{Conclusions}

The CLIC Test Facility CTF3 conducted a rich experimental program, addressing various aspects of the accelerator technology needed for the CLIC concept and solving the vast majority of issues related to drive beam generation, power production and two-beam acceleration. In particular, high-gradient acceleration beyond $100 \mathrm{MV} / \mathrm{m}$ using X-band room temperature is now well established, as well as the production and use of a high-current drive beam as an efficient and reliable source of X-band RF power in the range of hundreds of MWs. CTF3 successfully completed its experimental program in December 2016 as planned, and stopped operation. The approval of the new CLEAR [31] program, using the CALIFES linac, will give the opportunity to maintain some local testing capability at CERN for CLIC instrumentation and high-gradient structure testing with beam, alongside with other non-CLIC-related accelerator R\&D. 


\section{ACKNOWLEDGEMENTS}

This paper documents, surely in a largely incomplete manner, over more than a decade long collective effort of a large collaboration. The authors wish to acknowledge all individuals who participated over this period to the conception, design, construction, commissioning and operation of the CLIC Test Facility, CTF3.

\section{References}

[1] G. Geschonke and A. Ghigo Eds, "CTF3 Design Report", CERN, Geneva, Switzerland, CERN/PS 2002-008 (RF).

[2] M. Aicheler et al., "A Multi-TeV Linear Collider Based on CLIC Technology - CLIC Conceptual Design Report", CERN, Geneva, Switzerland, CERN-2012-007; SLAC-R-985; KEK-Report-2012-1; PSI-12-01; JAI-2012-001.

[3] Y. Thiery, J. Gao, J. LeDuff, "CTF3 Drive-Beam Injector Design", in Proc. 20th International Linac Conf., Monterey, CA, USA, August 2000, pp. 95-97.

[4] P. Urschutz et al., "Beam Dynamics Studies and Emittance Optimization in the CTF3 Linac at CERN", in Proc. EPAC 2006, Edinburgh, UK, June 2006, pp. 798-800.

[5] A. Dabrowski et al. "Measuring the Bunch Frequency Multiplication at CTF3" in Proc. 1st International Particle Accelerator Conf., Kyoto, Japan, May 2010, pp. 1107-1109.

[6] P. Urschutz et al., "Beam Dynamics and First Operation of the Sub-Harmonic Bunching System in the CTF3 Injector", in Proc. EPAC 2006, Edinburgh, UK, June 2006, pp. 771-773.

[7] E. Jensen, "CTF3 Drive Beam Accelerating Structures", in Proc. 21st International Linac Conf., Gyeongju, Korea, August 2002, pp. 34-36.

[8] R. Corsini et al. "First Full Beam Loading Operation with the CTF3 Linac" in Proc. 9th European Particle Accelerator Conf., Lucerne, Switzerland, July 2004, pp. 39-41.

[9] P. Urschu?tz et al. "Efficient Long-Pulse Fully Loaded CTF3 Linac Operation" in Proc. 2006 Linear Accelerator Conf., Knoxville, Tennessee, USA, August 2006, pp. 31-33.

[10] D. Alesini et al., "Beam instability induced by RF deflectors in the combiner ring of the CLIC test facility and mitigation by damped deflecting structures", Phys. Rev. ST Accel. Beams, vol. 14, p. 022001 (2011).

[11] R. Corsini et al., "CTF3 Status and Plans", ICFA Beam Dynamics Newsletter, vol. 62, p. 165 (2013).

[12] D. Schulte et al., "Status Of The CLIC Phase and Amplitude Stabilisation Concept", in Proc. 25th Linear Accelerator Conf., Tsukuba, Japan, September 2010, pp. 103-105.

[13] G. Sterbini, "Beam current stability in CTF3", presented at the International Workshop on Future Linear Colliders, Geneva, Switzerland (2010), http://agenda.linearcollider.org/event/4507/contributions/17550/attachments/14172/23264/CTF3BeamCurrentStability.pdf

[14] P.K. Skowronski et al., "Status and Plans for Completion of the Experimental Programme of the CLIC Test Facility CTF3", in Proc. IPAC 2016, Busan, Korea, May 2016, paper THPMB046, pp. 3347 3350 .

[15] L. Malina et al., "Recent Improvements in Drive Beam Stability in CTF3", in Proc. IPAC 2016, Busan, Korea, May 2016, paper WEPOR007, pp. 2677 - 2680. 
[16] J. Roberts et al., "Demonstration of CLIC Level Phase Stability using a High Bandwidth, Low Latency Drive Beam Phase Feedforward System at the CLIC Test Facility CTF3", in Proc. IPAC 2016, Busan, Korea, May 2016, paper WEPOR006, pp. 2673 - 2676.

[17] J. Roberts? and A. Ghigo Eds, "CTF3 Design Report", CERN, Geneva, Switzerland, CERN/PS 2002-008 (RF).

[18] P.K. Skowronski et al., "The CLIC Feasibility Demonstration in CTF3", in Proc. IPAC 2011, San Sebastian, Spain, September 2011, pp. 1042-1044.

[19] I. Syratchev et al., "High Power Operation with Beam of a CLIC PETS Equipped with On/Off Mechanism", in Proc. IPAC 2012, New Orleans, Louisiana, USA, pp. 1852-1854.

[20] F. Peauger et al., "Status of the CTF3 Probe Beam Linac CALIFES", in Proc. 24th Linear Accelerator Conf., Victoria, British Columbia, Canada, September-October 2008, pp. 389 - 391.

[21] A. Palaia et al., "Effects of RF breakdown on the beam in the Compact Linear Collider prototype accelerator structure", Phys. Rev. ST Accel. Beams, vol. 16, 081004 (2013).

[22] R. Lillestol, "Power production experiments at the Test Beam Line in the CLIC Test Facility 3", Ph.D. thesis, Norwegian University of Science and Technology, Trondheim, Norway, 2010, CERN-THESIS-2010-246.

[23] E. Senes et al., "Results of the Beam-Loading Breakdown Rate Experiment at the CLIC Test Facility CTF3", presented at IPAC'17, Copenhagen, Denmark, May 2017, paper TUPAB017, this conference.

[24] J.L. Navarro Quirante et al., "CALIFES: A Multi-Purpose Electron Beam for Accelerator Technology Tests", in Proc. of LINAC 2014, Geneva, Switzerland, August - September 2014, paper MOPP030, pp. $121-123$.

[25] R. Lillestol et al., "Status of wakefield monitor experiments at the CLIC Test Facility", in Proc. IPAC 2016, Busan, Korea, May 2016, paper WEOBB02, pp. 3347 - 3350.

[26] A. Benot-Morell et al., "Beam Tests of the Prototype Beam Position Monitoring System for the Drive Beam Line of the CLIC Two-Beam Module at CTF3", in Proc. IPAC 2016, Busan, Korea, May 2016, paper MOPMR019, pp. 3347 - 3350.

[27] J.R. Towler et al., "Development and Test of High Resolution Cavity BPMs for the CLIC Main Beam Linac", in Proc. IBIC15, Melbourne, Australia, September 2015, paper TUPB060, pp. 475 - 478.

[28] M. Kastriotou, et al., "BLM crosstalk studies on the CLIC Two-Beam Module", in Proc. IBIC15, Melbourne, Australia, September 2015, paper MOPB045, pp. 148 - 151.

[29] E. Nebot Del Busto, et al., "Position resolution of optical fibre-based beam loss monitors using long electron pulses", in Proc. IBIC15, Melbourne, Australia, September 2015, paper WEBLA03, pp. 580 584.

[30] R. Kieffer et al., "Development of a Versatile OTR-ODR Station for Future Linear Colliders", in Proc. IBIC15, Melbourne, Australia, September 2015, paper TUPB057, pp. 461 - 464.

[31] http://clear.web.cern.ch 